\title{
$\bullet$ A Questionnaire Survey on Usage of Aerosol Boxes: An Indian perspective
}

\section{IJCRR}

Section: Healthcare

ISI Impact Factor

(2019-20): 1.628

IC Value (2019): 90.81

$\operatorname{SJIF}(2020)=7.893$

\section{Akhya Kumar Kar ${ }^{1}$, Ayya Syama Sundar ${ }^{2}$}

'Assistant Professor, Department of Anaesthesiology and Critical Care, Nizam's Institute of Medical Sciences, Panjagutta, Hyderabad, India; ${ }^{2}$ Additional Professor, Department of Anaesthesiology, AllMS, Bibinagar, Hyderabad, Telangana 5008162, India.

\section{ABSTRACT}

Introduction: The barrier method is one of the most effective methods to control the spread of infectious diseases.

Objectives: Intubation and extubation are aerosol-generating procedures and to safeguard from getting the infection in a pandemic situation like SARS-COVID19, the aerosol boxes are popular among health care workers. This questionnaire survey was carried out on the anesthesiologist resident doctors working in various medical colleges across India to identify the views of front-line professionals' on the worth of the aerosol boxes during various procedures.

Methods: This survey was conducted on 153 respondents with asking 25 questions regarding various usage of aerosol boxes related to intubation, extubation and sterilization. Gender and experience of more than 10 intubations were sought to find out whether it has any influence on the overall decision making. Statistical analysis was done with SPSS, chi-square tests used for categorical variables.

Results: Out of 153 participants $64.5 \%$ of respondents were female and $35.5 \%$ were males. $49.3 \%$ had more than 10 intubation experience with the boxes. Mask ventilation was difficult for $91 \%$ and intubation was difficult for $98 \%$ of respondents. $90 \%$ of respondents were against the use of the box for difficult intubations. More experienced one was in favour of selectively removing the mask while intubation $(P<0.001)$. Use of boxes for extubation and in ICU was better among experienced with $P<0.001$ and $\mathrm{P}=0.010$ respectively. Patient contamination (54\%) and inadequate sterilization (55\%) of boxes believed by many as problems related to its use.

Conclusions: Aerosol boxes are used by good numbers for mask ventilation and easy intubation, the experienced ones were more comfortable in using them for extubation and ICU procedures, however, sterilization and contamination is an issue to deal with.

Keywords: Intubation, Tracheal Extubation, SARS Coronavirus, Aerosol containment box, Aerosol generation

\section{INTRODUCTION}

The barrier method is one of the most effective methods to control the spread of infectious diseases. Face shields, masks, respirators, hand gloves, leg covers, personal protective equipment (PPE), even isolation chambers act as a barrier to non-infectious individuals from getting infected. Continuous use of such form of barriers useful to protect from getting infected but simple barriers like masks, shields, PPE are disposable which cause much environmental pollution and isolating critical patients not always possible. Anaesthesiologists are at risk as there is a high possibility of breach of the barrier while doing high-risk aerosol-generating procedures from the close appropriation of patients like intubation and extubation. With the current pandemic of SARS-COVID19 it's assumed that the viral load plays a significant role in getting a severe infection, ${ }^{1}$ physical barrier in the form of aerosol boxes gained much importance (FIGURE-1) for doing aerosol-generating procedure. The first of its kind was described by a Taiwanese doctor. ${ }^{2}$ The average dimension of those aerosol boxes are being $52 * 52$ which fits the head end of patients on the operating table. The box has 2 openings towards the anaesthesia machine end for the operator's hand insertion and another circular opening in the side for assistance. With gaining popularity over the boxes many modifications attempted over the past months with regard to the changing the shape of the opening, location of the opening, creating additional hole for assistance, changing the material

\section{Corresponding Author:}

Dr. Ayya Syama Sundar, Additional Professor, Department of Anaesthesiology, AIIMS, Bibinagar, Hyderabad, Telangana 508162, India. Contact: 8179309677; Email: sasyasyama@gmail.com

ISSN: 2231-2196 (Print) ISSN: 0975-5241 (Online)

Received: $22.09 .2020 \quad$ Revised: 17.11 .2020

Accepted: 23.12 .2020

Published: 25.04 .2021 
for disposable use, sloping of the top part of the transparent glass for better visibility and was effective to varying extents in addressing the short comings of the initial design. ${ }^{3-6} \mathrm{How}-$ ever, the aerosol boxes have many limitations too. As these are new things to adapt it takes some time to get skilled to do safe procedures with this box. Delay in airway management is common with these boxes making the patient prone to desaturation during the induction phase of anaesthesia. ${ }^{\text {? }}$ The visibility is an issue with fogging of head shields, spectacles and the refractory error it adds to the problems. Difficult hand-eye coordination increases the user inconvenience as the hand movement is limited inside the boxes. Alignment of the head for intubation is also an issue. The aerosol boxes were thought to decrease the use of PPE which were scanty at the time these boxes started in use however contamination of the head end of the patient is a real problem with the use of it. With the difficulties of airway management with boxes and limited space inside the box area, more patient parts in the head-end, come to contact with the anesthesiologist, contaminating the surface. Aerosol boxes are thought to help to reduce the spread of aerosol during extubation, their use has never been evaluated. Acceptance of the aerosol box for intubation in ICU is uncertain due to added challenges of managing critical patients and set up as well. With a limited no of randomised control trials, we thought a questionnaire would be the best guide at present regarding the usefulness and shortcomings of the aerosol boxes. The primary objective of the study is to collect the majoritarian view concerning the use of aerosol boxes and problems associated with them based on their personal experience.

\section{MATERIALS AND METHODS}

This survey was undertaken among anaesthesiologist resident doctors in various medical colleges across India. As it is a questionnaire-based survey, ethical approval was not required. The survey group were residents with experience of more than 2 years of experience. For clarity of thought and understanding, while answering, the questions were framed in simple terms. The replies were sought with google forms sent to individuals through their mobile contact no. To prevent bias while answering the messages, they were asked not to communicate with anyone else regarding these questions before filling in the suitable replies. The answers were converted to spreadsheets and graphs and verified for the results.

A total of 25 questions was posted to anaesthesia postgraduate resident doctors across various medical colleges in India. Each question had multiple choices out of only one answer supposed to select. Some questions were framed in such a way that, multiple answers possible however it was not allowed to do so with an intent to find the majoritarian view. Years of experience in anaesthesia, age and gender were asked in the questionnaire to identify their influence if any on the overall decision making. Less than 10 intubation attempts with the aerosol boxes are placed in a separate category as their answers may not represent the true outcome. Out of the respondents' experience, they were asked to comment on gross suggestions regarding the aerosol box use. Safety of using the aerosol boxes being carried out by asking for frequency of desaturation or complication incidences while managing the airway. Using the aerosol boxes for extubation and use in ICU were specifically sought to find out views regarding its usefulness.

The sample size was calculated using Statistical Software G Power 3.1.9.2. We Calculated the no of resident doctors being approximately 6000 based on the admissions and assumed $50 \%$ of them either have availability or have done at least 5 incidences of airway management with aerosol box. With a power of $80 \%$ and an alpha error of 0.05 and considering a moderate effect size of 0.55 , the sample size was calculated to be 143 . However, considering the possibility of improper entries 150 entries were sought.

Statistical analysis was performed using IBM SPSS (version 20 , IBM, I. The categorical variables were expressed as frequencies with percentages. The Chi-square test was used to assess the distribution of observed variables. $\mathrm{P}<0.05$ was considered statistically significant.

\section{RESULTS}

A total of 153 respondents took part in the survey. Out of which $64.5 \%$ were female and $35.5 \%$ were male. Respondents with experience of 10 or more intubations with aerosol box constituted $49.3 \%$. With strict institutional guideline, a significant no of residents had exposure to more intubations with the aerosol box $(\mathrm{P}=0.005)$. A total of $38.2 \%$ of respondents believed that the aerosol boxes useful in protecting against the spread of the virus while others either not sure or not convinced regarding its worth. Despite the view of the difficulty in mask ventilation $(91 \%)$ and intubation $(98 \%)$ at some point of the procedure, $71 \%$ recommend using the box for mask ventilation and $77 \%$ were in favour to use it for easy intubation. However, for difficult intubation, there seems to be a consensus among $90 \%$ of respondents against using the aerosol boxes (Figure 2). Significant female respondents (FIGURE-3) were against the use of aerosol boxes for difficult intubation situations $(\mathrm{P}=0.001)$ as compared to males. A total of $68 \%$ of respondents had experienced patient desaturation during intubation at some point in time. Crisis needing for frequent removal of the box for intubation was experienced by $18 \%$ of respondents (Table $1)$, whereas removal was significantly rare in male residents $(\mathrm{P}=0.03)$. Limited hand movement inside the box $(38.2 \%)$ and visibility (32.9\%) were considered as a major reason for difficult airway situation, however, the former was found to 
be a majoritarian view with less experience than the latter, (Table 2) which was the common view of the more experienced respondents with more than 10 intubation attempts $(\mathrm{P}=0.007)$. Early fatigue was a complaint by $44.6 \%$ of the respondents with $27 \%$ complained so in occasional situations. Probably due to the difficulties in airway management $52 \%$ of respondents were in favour to use the box while mask ventilation and electively remove it while intubation (Table 3 ). This opinion was significant among the most experienced ones rather than the other group with having lesser experience $(\mathrm{P}<0.0001)$. Many believed the inside box area was space-constrained (73\%), and high chances of contamination, add $55 \%$ of respondents either believed boxes were not sterilized or did not have a clue whether done so. A total of $73 \%$ believed boxes useful for extubation and proportionately used too $(68 \%)$, however, $31 \%$ of respondents experienced removal of the box during extubation at some point to manage airway related complications which are also reflected in $26 \%$ believing that in fact, extubation complications increased with using the aerosol boxes (Figure 4). The result for ICU intubations was also not encouraging with $42 \%$ of respondents not using it, (Figure 5) and the major reason being the emergency airway situation gives very little time to use the boxes $(55 \%)$.

\section{DISCUSSION}

Our survey tried to evaluate individual belief and difficulties faced by resident doctors while adapting to the new skill of airway management with using the aerosol box. Almost $50 \%$ of residents still are not enough experienced with using the boxes, probably that is related to liberal institutional guidelines and individual assumption regarding worth of using it. The unanimous view of difficulty in intubation, along with constraint of space, limited hand movement played a role in less no of residents using it. Studies have proven intubation takes significantly higher time with using the intubation boxes with first pass success with aerosol boxes use was $75-83 \%$, which could be one of the reason of high incidence of desaturation and urgent removal of the box among the patients while intubation. ${ }^{7}$ Less experienced ones had major complain of limited hand movement issues while more experienced ones seem to adapt to the changes well, however, visibility was an issue in the latter (Table 2 ). With the use of face shields, safety goggles, masks and many wearing glasses, the boxes add another barrier, causing refractory changes to visibility as well as fogging creates enough discomfort while managing the airway. With the increase in intubation time and uncomfortable hand positions, fatigue of fingers was noted as a major cause of limited acceptance of the aerosol boxes. With mask ventilation being an aerosol-generating procedure and intubation is done in a controlled manner with the adequate effect of paralytic agents,${ }^{8}$ significant number of respondents (Table 3) with more than 10 intubation experience were in favour of removing the box electively while intubation after the initial mask ventilation with it as compared to respondents with less experience $(\mathrm{P}<0.001)$. Limited space inside the box area while intubation and difficult hand movements make the inside box area prone to contamination, which negates the actual purpose of the introduction of the boxes. The virus survives on plastic for 6-7hrs, so an unsterilized box or contaminated patient coverings can be a potential source of infection. ${ }^{9}$ Studies have proven with the aerosol boxes, the inside area of the transparent acrylic is contaminated with the aerosol particles ${ }^{10}$ however many respondents were sceptical about the adequacy of sterilization of the boxes after their use, which makes them a prominent infective source rather than giving protection. Probably for the same reason, adverse opinion exists with regards to the use of rigid reusable barriers. ${ }^{11}$ Extubation is a significant moment to generate aerosol due to the cough related to emergence from anaesthesia and irritation of endotracheal tube. Good no of respondents believed the aerosol boxes useful for extubation $(71 \%)$ the experienced ones were significantly better users as compared to less experienced ones. (Table 6). A high no of respondents (31\%) faced the situation of removal of boxes while extubation which is again significantly less with those experienced $(\mathrm{P}<0.001)$. Airway complications are 3 times more common during extubation than during intubation, ${ }^{12}$ and with the use of aerosol boxes, the risk is manifold in facing undesirable situations during extubation. Airway procedures in ICU need special mention as the COVID related lung damage make the patients left with very limited or no lung reserve with most patients oxygenated with non-invasive ventilation or high flow nasal oxygen therapy. With worse outcome among mechanically ventilated patients with COVID related lung damage, patients are referred to intubate, late in the course of the disease. ${ }^{13}$ Due to all the above factors, intubation in ICU, are of grave emergency with very limited time to react and do the procedure. That makes the use of aerosol boxes in ICU very challenging. Probably that's reflected in significant no of respondents (42\%) not using the boxes for airway management in ICU, however the experienced ones the acceptance is significantly better $(\mathrm{P}=0.010)$.

The limitations of our survey include, majority of respondents are less experienced with airway management with the aerosol box, which is also of not uniform type everywhere with some modification based on the preference. There was no uniformity in strict institutional guideline regarding its use in many of the respondents. A comparison of airway management with and without box was not done. Also, the questions were subjective which leaves scope for future research to objectively assess the outcome. 


\section{CONCLUSION}

Aerosol boxes are believed to be effective in curbing the spread of the virus to health care workers. Effective use is accepted in the form of mask ventilation, easy intubations. In difficult intubation cases, its use is not preferred. Cleaning the boxes after the procedure is an issue to address. Usage of the box for extubation is a preferred modality to limit the spread of aerosol, which need some experience to adapt. The ICU setting where the need to use the box is most being used least.

\section{ACKNOWLEDGEMENT}

Authors acknowledge the immense help received from the scholars whose articles are cited and included in references of this manuscript.

\section{Conflict of Interest: None}

Financial support: Nil

\section{REFERENCES}

1. Zheng S, Fan J, Yu F. Viral load dynamics and disease severity in patients infected with SARS-CoV-2 in Zhejiang province, China, January-March 2020: retrospective cohort study: BMJ 2020;369:1443.

2. Everington K. Taiwanese doctor invents device to protect US doctors against coronavirus. 2020. https://www.taiwannews. com.tw/en/news/3902435 (accessed 28/04/2020).
3. Asokan K, Babu B, Jayadevan A. Barrier enclosure for airway management in COVID-19 pandemic. Indian J Anaesth 2020;64:153-154.

4. Singh B, Singla SL, Gulia P. Aerosol containment device for use on suspected COVID-19 patients. Indian J Anaesth 2020;64:154156.

5. Gupta V, Sahani A, Mohan B et al. Negative pressure aerosol containment box: An innovation to reduce COVID-19 infection risk in healthcare workers. J Anaesthesiol Clin Pharmacol 2020;36:144-147.

6. Vijayaraghavan S, Puthenveettil N. Aerosol box for protection during airway manipulation in covid-19 patients. Indian J Anaesth 2020;64:148-149.

7. Begley JL, Lavery KE, Nickson CP, et al. The aerosol box for intubation in coronavirus disease 2019 patients: an in-situ simulation crossover study: Anaesthesia 2020;75:1014-1021.

8. Brewster DJ, Chrimes N, Do TB, et al. Consensus statement: Safe airway society principles of airway management and tracheal intubation specific to the COVID-19 adult patient group. Med J 2020;212:472-481.

9. Chin AWH, Chu JTS, Perera MRA, et al. Stability of SARS-CoV-2 in different environmental conditions. medRxiv 2020. doi: https://doi.org/10.1016/ S2666-5247(20)30003-3.

10. Matava CT, Yu J, Denning S: Clear plastic drapes may be effective at limiting aerosolization and droplet spray during extubation: Implications for COVID-19. Can J Anaesth 2020;67(7):902-904.

11. Ortega R, Nozari A, Canelli R. More on barrier enclosure during endotracheal intubation. N Engl J Med 2020:382.

12. Asai T, Koga K, Vaughan RS. Respiratory complications associated with tracheal intubation and extubation. Br J Anaesth 1998;80:767-775.

13. Yang X, Yu Y, Xu J, et al. Clinical course and outcomes of critically ill patients with SARS-CoV-2 pneumonia in Wuhan, China: a single-centred, retrospective, observational study. Lancet Respir Med 2020;8(5):475-481.

Table 1: Incidence of emergency removal of the box while intubation

\begin{tabular}{lccc} 
GENDER & Rarely & Sometimes & P-Value \\
Female & 14 & 82 & \\
Male & 16 & 38 & 0.03 \\
\hline
\end{tabular}

Table 2: Reason for difficulty in airway management

\begin{tabular}{lccccc} 
& Alignment & Limited movement & Positioning & Visibility & P-value \\
$<10$ no Intubation with box & 16 & 36 & 8 & 16 & 0.007 \\
$>10$ no of intubation with box & 12 & 22 & 8 & 32 & \\
\hline
\end{tabular}

Table 3: Recommendation regarding elective removal of the box after mask ventilation for intubation

\begin{tabular}{llll} 
& No & Yes & P-Value \\
$<10$ no Intubation with box & 50 & 26 & $<0.0001$ \\
$>10$ no of intubation with box & 22 & 52 & $<$ \\
\hline
\end{tabular}




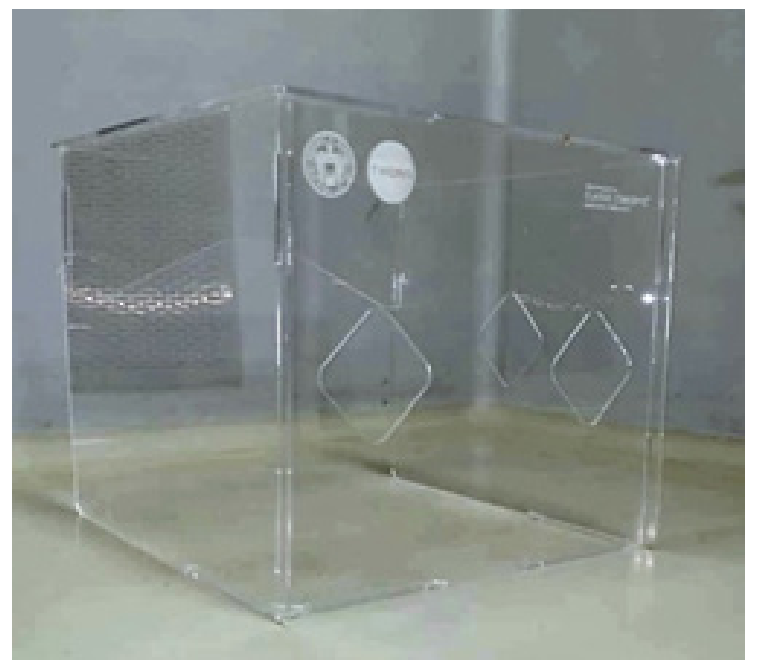

Figure 1: Aerosol Box.

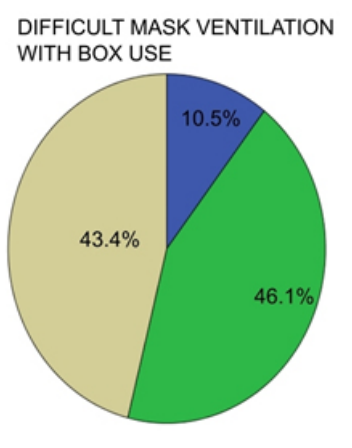

DIFFICULT INTUBATION WITH BOX USE

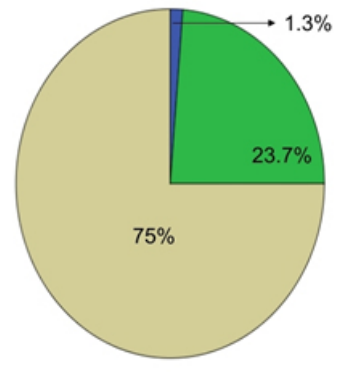

YES

NO

SOMETIMES

Figure 2a: Difficulties in procedures with aerosol box use.

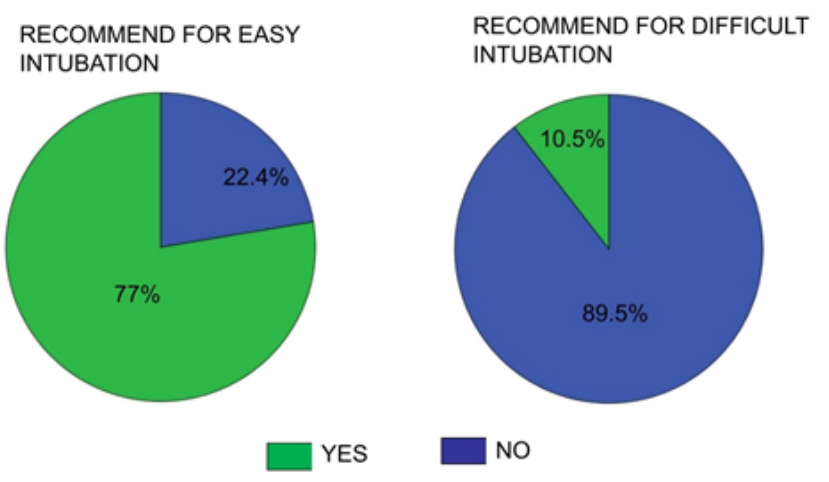

Figure 2b: Respondents view on the recommendation of Aerosol box use.
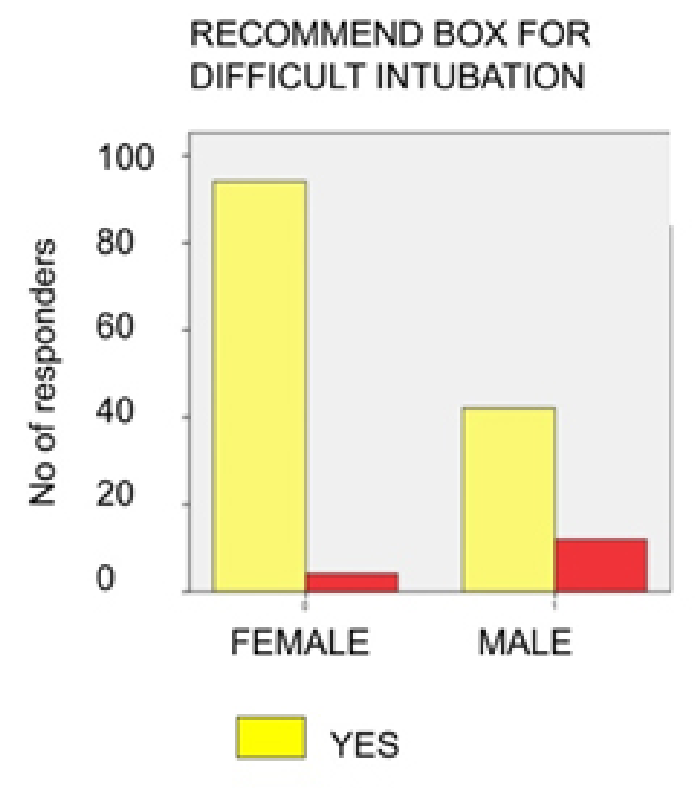

NO

Figure 3: Gender differentiation on Aerosol box use for difficult intubation.

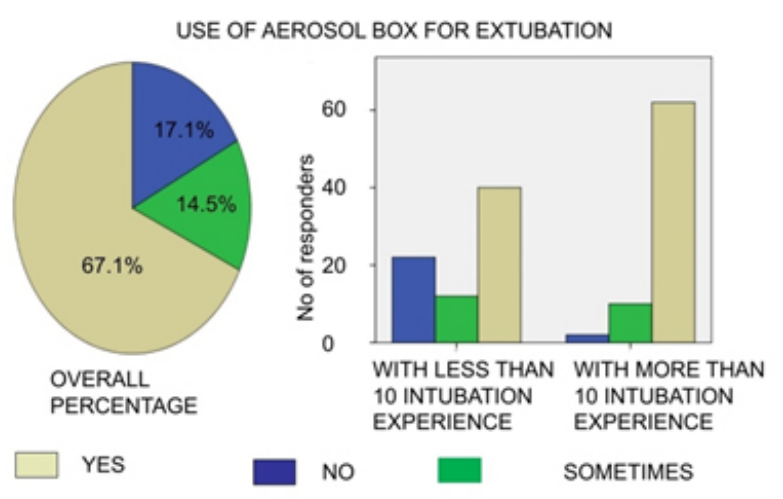

Figure 4: Aerosol Box use for Extubation.

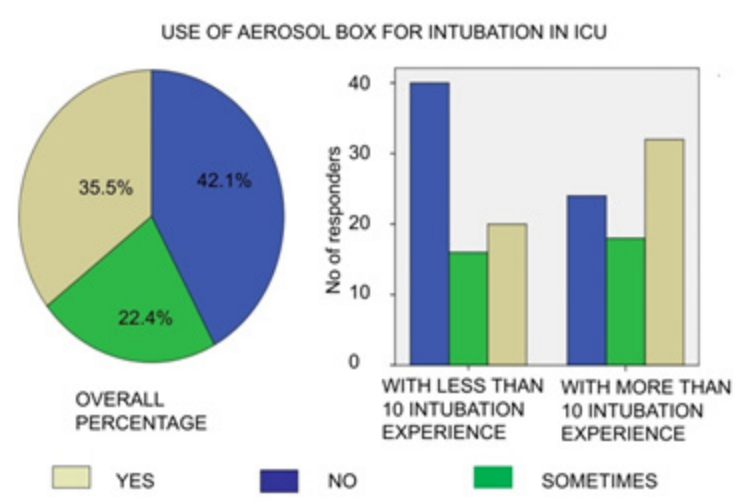

Figure 5: Aerosol box use in ICU. 